\title{
Radiation length imaging with high resolution telescopes
}

\author{
Ulf Stolzenberg ${ }^{* a}$, Benjamin Schwenker ${ }^{a}$, Ariane Frey ${ }^{a}$, Philipp Wieduwilt ${ }^{a}$, Carlos \\ Marinas $^{b}$, Florian Lütticke ${ }^{b}$, Martin Ritter ${ }^{c}$ and Waleed Ahmed ${ }^{d}$ \\ ${ }^{a}$ II. Physikalisches Institut, Universität Göttingen, Germany \\ ${ }^{b}$ Physikalisches Institut, Universität Bonn, Germany \\ ${ }^{c}$ Faculty of Physics, Ludwig-Maximilians-Universität München, Germany \\ ${ }^{d}$ Department of Physics, McGill University, Montreal, Canada \\ E-mail: ulf.stolzenberg@phys .uni-goettingen.de, \\ Benjamin.Schwenker@phys.uni-goettingen.de, ariane.frey@cern.ch, \\ philipp.wieduwiltastud.uni-goettingen.de, cmarinaseuni-bonn.de, \\ luetticke@physik.uni-bonn.de, martin.ritterelmu.de, \\ waleed.ahmed2@mail.mcgill.ca
}

\begin{abstract}
The construction of low mass vertex detectors with a high level of system integration is of great interest for next generation collider experiments. Radiation length images with a sufficient spatial resolution can be used to measure and disentangle complex radiation length $\left(X / X_{0}\right)$ profiles and contribute to the understanding of vertex detector systems. Beam test experiments with multi $\mathrm{GeV}$ particle beams and high resolution tracking telescopes provide an opportunity to obtain precise $2 \mathrm{D}$ images of the radiation length of thin planar objects. At the heart of the $X / X_{0}$ imaging is a spatially resolved measurement of the scattering angles of particles traversing the object under study.

In order to demonstrate the capabilities of $X / X_{0}$ imaging, a beam test experiment has been conducted. The device under test was a mechanical prototype half-ladder of the Belle II pixel detector (PXD). The measured $X / X_{0}$ image was overlaid with a Geant 4 simulation of a PXD half-ladder and excellent agreement was found. As shown here, radiation length imaging of detectors can serve as a method to validate geometry models of complex vertex detectors.
\end{abstract}

38th International Conference on High Energy Physics

3-10 August 2016

Chicago, USA

${ }^{*}$ Speaker. 


\section{Introduction}

Physics requirements limit the mass of vertex and tracking detectors for next generation collider experiments [1]. To meet the conflicting goals of minimizing detector mass and also incorporating cooling, power distribution and readout electronics an integrated system design approach is needed. Material effects like multiple scattering degrade the precision of the reconstructed vertex position and track parameters. In the case of multiple scattering, the magnitude of the effect depends on the local material budget measured in units of the material constant $X_{0}$. It is therefore crucial to carefully limit and account for every material contribution in the detector acceptance region.

In order to study the material composition of planar objects like for example modules of the Belle II pixel detector (PXD) a method has been developed to measure high resolution 2D images of the radiation length $X / X_{0}$. The radiation length of a planar object can be extracted by fitting the distribution of deflection angles $\vartheta$ of many traversing particles, which can be reconstructed with a high resolution telescope. The spatial resolution of the telescope opens the possibility to measure the radiation length in small planar areas on the device under study.

\section{Radiation length imaging}

The radiation length measurement method is described in [2]. The basic idea is to reconstruct multiple scattering angles from charged particle tracks on a central measurement plane. A Kalman filter pair is employed to estimate the local track state and covariance matrix before the scattering $\left(\eta^{\text {in }}, C^{\text {in }}\right)$ from the upstream hits and the state and covariance matrix after the scattering $\left(\eta^{\text {out }}, C^{\text {out }}\right)$ from the downstream hits. Scattering angles can then be calculated as the difference between the local track slopes. The particle intersection point is the average of the upstream and downstream track intersections.

The scattering angles of a set of charged particle tracks are sorted into small pixels according to their intersection with the measurement plane. In order to compute the radiation length value of the material in one pixel of the image the measured angle distribution of this pixel is fitted by the model

$$
f_{\text {reco }}(\vartheta)=\mathrm{f}_{\text {MSC }} \otimes \frac{1}{\left(\lambda \sigma_{\text {reso }}\right) \sqrt{2 \pi}} \exp \left(\frac{-\vartheta^{2}}{2\left(\lambda \sigma_{\text {reso }}\right)^{2}}\right)
$$

The function $\mathrm{f}_{\mathrm{MSC}}$ corresponds to the multiple scattering distribution, which is described by a theoretical model, for example introduced by Highland [3]. The width of the angle distribution is dependent on the ratio $X / \mathrm{X}_{0}$, where $X$ is the material thickness and $X_{0}$ is the radiation length constant of the material. Formulas for calculating $X_{0}$ from material properties can be found in [4]. The second part of eq. 2.1 corresponds to measurement errors of the deflection angles, due to the finite angle resolution of the telescope $\sigma_{\text {reso }}$. The value of $\sigma_{\text {reso }}$ can be calculated from $C^{\text {in }}$ and $C^{\text {out }}$ via error propagation. The correction factor $\lambda$ is used to account for a possible bias in $\sigma_{\text {reso }}$ and must be extracted from a dedicated calibration measurement on a planar object with a well known material distribution. The telescope resolution $\sigma_{\text {reso }}$ is independent of the material distribution on the device under study and therefore the correction factor $\lambda$ is valid for all measurements with the same telescope setup. 


\section{Beam test results and Geant4 simulations}

The $X / X_{0}$ measurements presented here were performed using a $4 \mathrm{GeV}$ electron beam at DESY. The measurements were conducted with the AIDA telescope [5] with spacings of approximately $40 \mathrm{~mm}$. On the measurement plane the intersection position error was found to be roughly $3 \mu \mathrm{m}$ and the calibrated angle resolution $\lambda \cdot \sigma_{\text {reso }}$ was approximately $150 \mu \mathrm{rad}$. The software for the reconstruction of the beam test data can be found at [6].

As a complement to the beam test measurements Geant 4 simulations [7] of the PXD half-ladder can be performed with the Belle II software framework. The simulation involved shooting Geantinos through the simulated half-ladder on a dense grid. Each Geantino registers all material contributions along its path and computes the total radiation length. This radiation length is assigned to the corresponding grid point and a $2 \mathrm{D}$ radiation length image is generated.
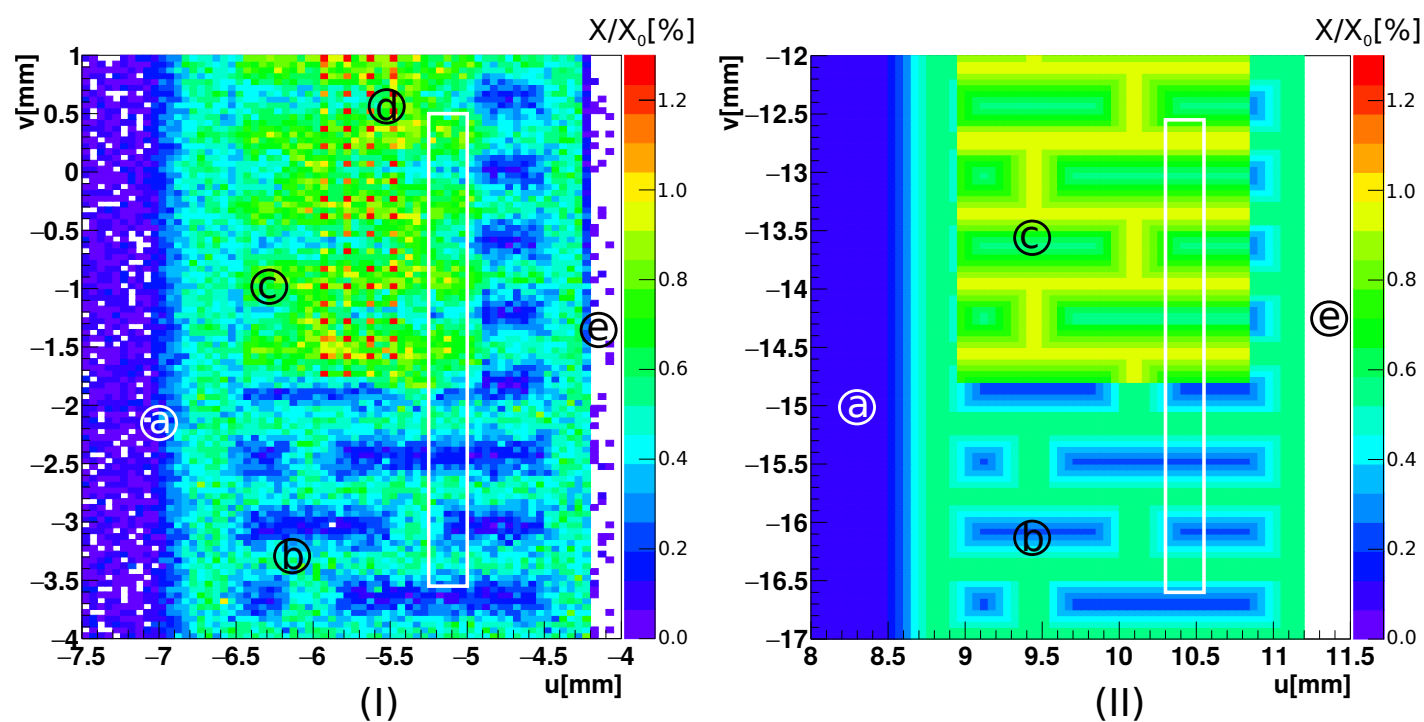

Figure 1: Beam test data image (I) and simulation image (II). Both images show the same region on a PXD module. Areas of different material contributions are indicated by the letters (a)-(e).

In Fig. $1 X / X_{0}$ images of an area on the Belle II PXD half-ladder are shown. Both images have a bin side length of $50 \mu \mathrm{m}$. The left image shows the results of the beam test measurements, while the picture on the right depicts image of the simulated half-ladder. Both images show the sensitive area (a) on the left, the silicon balcony with grooves (b) and an read-out ASIC (c) as well as air on the right hand side of the images (e). The beam test measurements were performed on a mechanical PXD sample, while the simulation image shows a final Belle II half-ladder design. The difference resides in the size and exact position of the ASICs on the balcony. Also the bump bonds, which are visible as a pattern of small red points in the beam test measurements (d), are not modeled in the Geant4 simulation.

In order to do a quantitative comparison between the two images profile projections are employed (indicated by the white boxes in Fig. 1). The profiles of both images are shown in Fig. 2. The cut of the profiles is going along the balcony across the edge of the ASIC. The profiles match each other well. The position of the edge of the ASIC is shifted by approximately $100 \mu \mathrm{m}$. Moreover an offset of approximately $0.1 \%$ of $X / X_{0}$ in the ASIC region is visible. This can be attributed to 


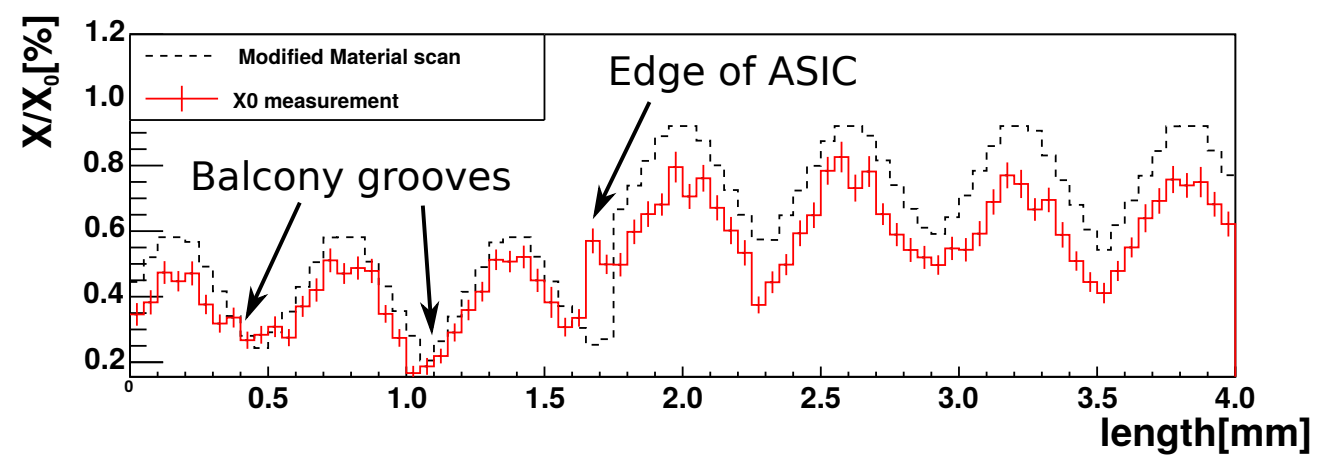

Figure 2: Radiation length profile of the simulation (dashed black line) and the beam test measurements (red) in the balcony region (see white boxes in Fig. 1)

the fact, that the measurements were performed on a mechanical prototype of the PXD half-ladder including ASICs, which were thinner than the ASICs of $300 \mu \mathrm{m}$ used in the simulations.

\section{Conclusion}

This work presents a method to measure and image the radiation length of thin planar objects in beam test environments. It is furthermore possible to compare these images quantitatively with the Geant 4 simulation of the detector. The comparison on the $50 \mu \mathrm{m}$ scale provides the opportunity to assess, validate and improve if necessary the geometry model in Geant 4 .

The measurements leading to these results have been performed at the Test Beam Facility at DESY Hamburg (Germany), a member of the Helmholtz Association (HGF). This work was supported by the German Ministerium für Bildung, Wissenschaft, Forschung und Technologie (BMBF) and the VolkswagenStiftung.

\section{References}

[1] W. E. Cooper, Low-Mass Materials and Vertex Detector Systems, in proceedings of Vertex2013, PoS (Vertex2013) 036 (2013)

[2] U. Stolzenberg et al., Radiation length imaging with high-resolution telescopes, to be published in Nucl. Instr. Meth. A, arXiv:1609.02402 (2016)

[3] V. Highland, Some practical remarks on multiple scattering, Nucl. Instr. and Meth. 129 (1975) 497-499

[4] C. Patrignani et al., Review of Particle Physics: Passage of particles through matter, Particle Data Group, Chin. Phys. C40, (2016)

[5] H. Jansen et al., Performance of the EUDET-type beam telescopes, doi:10.1140/epjti/s40485-016-0033-2 arXiv:1603.09669 [physics.ins-det]

[6] Test beam software framework, https://bitbucket.org/BenjaminSchwenker/tbsw (2016)

[7] J. Allison et al., Geant4 developments and applications, IEEE Transactions on Nuclear Science 53 (2006) 270-278 